\title{
The Quadruple-Tank Process: A Multivariable Laboratory Process with an Adjustable Zero
}

\author{
Karl Henrik Johansson, Member, IEEE
}

\begin{abstract}
A novel multivariable laboratory process that consists of four interconnected water tanks is presented. The linearized dynamics of the system have a multivariable zero that is possible to move along the real axis by changing a valve. The zero can be placed in both the left and the right half-plane. In this way the quadruple-tank process is ideal for illustrating many concepts in multivariable control, particularly performance limitations due to multivariable right half-plane zeros. The location and the direction of the zero have an appealing physical interpretation. Accurate models are derived from both physical and experimental data and decentralized control is demonstrated on the process.
\end{abstract}

Index Terms-Education, laboratory process, multivariable control, multivariable zeros.

\section{INTRODUCTION}

$\mathbf{M}$ ULTIVARIABLE control techniques have received increased industrial interest [31]. It is often hard to tell when these methods are needed for improved performance in practice and when simpler control structures are sufficient. A key issue is the functional limits of the system: what design specifications are reasonable? It was already pointed out by Bode [6] that nonminimum-phase characteristics of a system impose limitations for linear feedback designs. Still it happens that unrealistic specifications are made, as pointed out in [3], [11], and [33]. Performance limitations in control systems have received extensive interest recently. Several new results, particularly for scalar and multivariable linear systems, have been presented. Bode's original result together with extensions are covered in the textbooks [10], [30]. Zames and Francis [9], [36], [38] showed that right half-plane zeros impose restrictions on the sensitivity function: if the sensitivity is forced to be small in one frequency band, it has to be large in another, possibly yielding an overall bad performance. They also showed that if the system does not have any right half-plane zeros, then theoretically it can be arbitrarily well controlled. The latter result has been generalized to decentralized control structures [19], [20], [37].

This paper describes a new laboratory process, which was designed to illustrate performance limitations due to zero location in multivariable control systems. The process is called the quadruple-tank process and consists of four interconnected water tanks and two pumps. The system is shown in Fig. 1. Its

Manuscript received September 21, 1998; revised April 15, 1999. Recommended by Associate Editor, F. Doyle. This work was supported by the Swedish Research Council for Engineering Science under Contract 95-759.

The author is with the Department of Electrical Engineering and Computer Sciences, University of California, Berkeley, CA 94720 USA (e-mail: johans@eecs.berkeley.edu).

Publisher Item Identifier S 1063-6536(00)03195-X inputs are the voltages to the two pumps and the outputs are the water levels in the lower two tanks. The quadruple-tank process can easily be build by using two double-tank processes, which are standard processes in many control laboratories [4], [5]. The setup is thus simple, but still the process can illustrate several interesting multivariable phenomena. The linearized model of the quadruple-tank process has a multivariable zero, which can be located in either the left or the right half-plane by simply changing a valve. Both the location and the direction of a multivariable zero are important for control design. They have direct physical interpretations for the quadruple-tank process, which make the process suitable to use in control education.

Few multivariable laboratory processes have been reported in the literature. Mechanical systems such as the helicopter model [1], [24] and the active magnetic bearing process [35] have been developed at ETH, Zürich, Switzerland. Davison has developed a water tank process, where multivariable water level control and temperature-flow control can be investigated [8]. Some multivariable laboratory processes are commercially available, for example from Quanser Consulting in Canada, Educational Control Products in the United States, and Feedback Instruments and TecQuipment in the United Kingdom. There does not, however, seem to exist any laboratory processes that demonstrate multivariable zero location and direction in an illustrative way. This was one of the main motivations for the development of the quadruple-tank process [15].

The outline of this paper is as follows. A nonlinear model for the quadruple-tank process based on physical data is derived in Section II. It is linearized and the linear model is validated using experimental data. The location and the direction of a multivariable zero of the linearized model are derived in Section III. It is shown that the valve positions of the process uniquely determine if the system is minimum phase or nonminimum phase. The relative gain array is also derived. Section IV discusses the nonlinear model of the quadruple-tank process. Properties of the nonlinear zero dynamics and of the stationary point of the nonlinear model are given. In Section V linear models are estimated from experimental data and they are compared to the physical model. Decentralized proportional integral (PI) control of the quadruple-tank process is presented in Section VI, where performance losses due to right half-plane zeros are illustrated. Some concluding remarks are given in Section VII. Early versions of this paper have been presented as [15], [18].

\section{PHYSICAL MOdel}

In this section we derive a mathematical model for the quadruple-tank process from physical data. The section ends with some further comments on the construction of the process. 


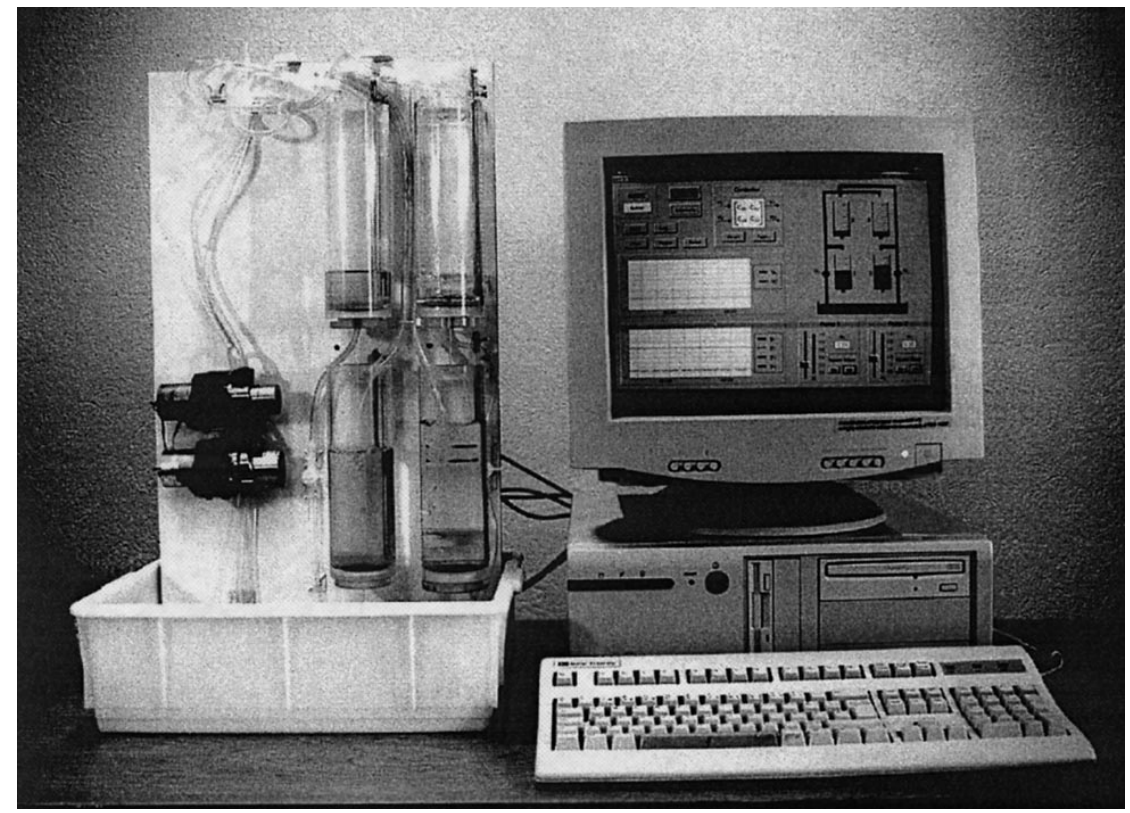

Fig. 1. The quadruple-tank process shown together with a controller interface running on a PC.

A schematic diagram of the process is shown in Fig. 2. The target is to control the level in the lower two tanks with two pumps. The process inputs are $v_{1}$ and $v_{2}$ (input voltages to the pumps) and the outputs are $y_{1}$ and $y_{2}$ (voltages from level measurement devices). Mass balances and Bernoulli's law yield

$$
\begin{aligned}
\frac{d h_{1}}{d t} & =-\frac{a_{1}}{A_{1}} \sqrt{2 g h_{1}}+\frac{a_{3}}{A_{1}} \sqrt{2 g h_{3}}+\frac{\gamma_{1} k_{1}}{A_{1}} v_{1} \\
\frac{d h_{2}}{d t} & =-\frac{a_{2}}{A_{2}} \sqrt{2 g h_{2}}+\frac{a_{4}}{A_{2}} \sqrt{2 g h_{4}}+\frac{\gamma_{2} k_{2}}{A_{2}} v_{2} \\
\frac{d h_{3}}{d t} & =-\frac{a_{3}}{A_{3}} \sqrt{2 g h_{3}}+\frac{\left(1-\gamma_{2}\right) k_{2}}{A_{3}} v_{2} \\
\frac{d h_{4}}{d t} & =-\frac{a_{4}}{A_{4}} \sqrt{2 g h_{4}}+\frac{\left(1-\gamma_{1}\right) k_{1}}{A_{4}} v_{1}
\end{aligned}
$$

where

$A_{i} \quad$ cross-section of Tank $i$;

$a_{i} \quad$ cross-section of the outlet hole;

$h_{i} \quad$ water level.

The voltage applied to Pump $i$ is $v_{i}$ and the corresponding flow is $k_{i} v_{i}$. The parameters $\gamma_{1}, \gamma_{2} \in(0,1)$ are determined from how the valves are set prior to an experiment. The flow to Tank 1 is $\gamma_{1} k_{1} v_{1}$ and the flow to Tank 4 is $\left(1-\gamma_{1}\right) k_{1} v_{1}$ and similarly for Tank 2 and Tank 3 . The acceleration of gravity is denoted $g$. The measured level signals are $k_{c} h_{1}$ and $k_{c} h_{2}$. The parameter values of the laboratory process are given in the following table:

$$
\begin{array}{llc}
A_{1}, A_{3} & {\left[\mathrm{~cm}^{2}\right]} & 28 \\
A_{2}, A_{4} & {\left[\mathrm{~cm}^{2}\right]} & 32 \\
a_{1}, a_{3} & {\left[\mathrm{~cm}^{2}\right]} & 0.071 \\
a_{2}, a_{4} & {\left[\mathrm{~cm}^{2}\right]} & 0.057 \\
k_{c} & {[\mathrm{~V} / \mathrm{cm}]} & 0.50 \\
g & {\left[\mathrm{~cm} / \mathrm{s}^{2}\right]} & 981 .
\end{array}
$$

The model and control of the quadruple-tank process are studied at two operating points: $P_{-}$at which the system will be shown to

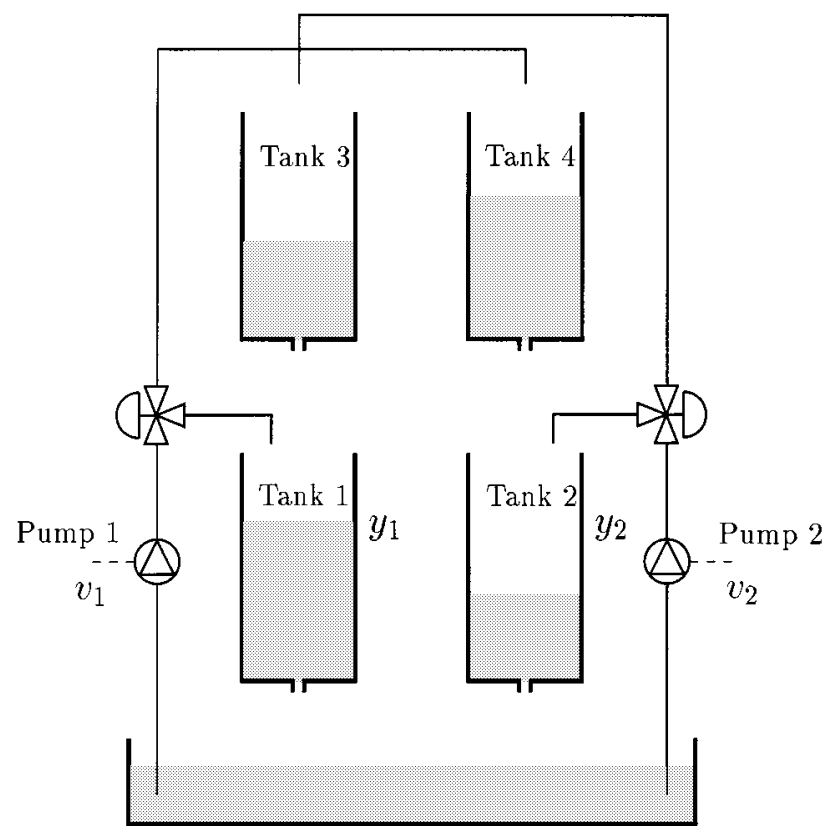

Fig. 2. Schematic diagram of the quadruple-tank process. The water levels in Tanks 1 and 2 are controlled by two pumps. The positions of the valves determine the location of a multivariable zero for the linearized model. The zero can be put in either the left or the right half-plane.

have minimum-phase characteristics and $P_{+}$at which it will be shown to have nonminimum-phase characteristics. The chosen operating points correspond to the following parameter values:

\begin{tabular}{llll} 
& & $P_{-}$ & $P_{+}$ \\
\hline$\left(h_{1}^{0}, h_{2}^{0}\right)$ & {$[\mathrm{cm}]$} & $(12.4,12.7)$ & $(12.6,13.0)$ \\
$\left(h_{3}^{0}, h_{4}^{0}\right)$ & {$[\mathrm{cm}]$} & $(1.8,1.4)$ & $(4.8,4.9)$ \\
$\left(v_{1}^{0}, v_{2}^{0}\right)$ & {$[\mathrm{V}]$} & $(3.00,3.00)$ & $(3.15,3.15)$ \\
$\left(k_{1}, k_{2}\right)$ & {$\left[\mathrm{cm}^{3} / \mathrm{Vs}\right]$} & $(3.33,3.35)$ & $(3.14,3.29)$ \\
$\left(\gamma_{1}, \gamma_{2}\right)$ & & $(0.70,0.60)$ & $(0.43,0.34)$
\end{tabular}



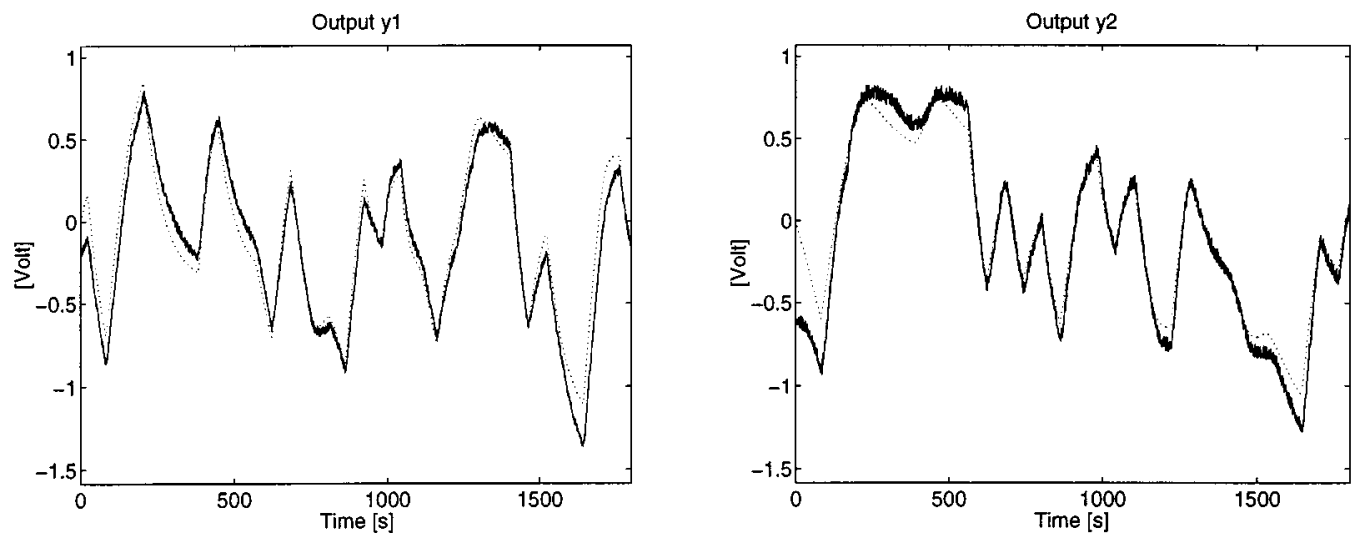

Fig. 3. Validation of the minimum-phase model $G_{-}$. The outputs from the model (dashed lines) together with the outputs from the real process (solid lines) are shown.

Introduce the variables $x_{i}:=h_{i}-h_{i}^{0}$ and $u_{i}:=v_{i}-v_{i}^{0}$. The linearized state-space equation is then given by

$$
\begin{aligned}
\frac{d x}{d t}= & {\left[\begin{array}{cccc}
-\frac{1}{T_{1}} & 0 & \frac{A_{3}}{A_{1} T_{3}} & 0 \\
0 & -\frac{1}{T_{2}} & 0 & \frac{A_{4}}{A_{2} T_{4}} \\
0 & 0 & -\frac{1}{T_{3}} & 0 \\
0 & 0 & 0 & -\frac{1}{T_{4}}
\end{array}\right] x } \\
& +\left[\begin{array}{ccc}
\frac{\gamma_{1} k_{1}}{A_{1}} & 0 \\
0 & \frac{\gamma_{2} k_{2}}{A_{2}} \\
0 & \frac{\left(1-\gamma_{2}\right) k_{2}}{A_{3}} \\
y= & 0 \\
\frac{\left(1-\gamma_{1}\right) k_{1}}{A_{4}} & 0 \\
0 & 0 & 0 \\
k_{c} & 0 & 0
\end{array}\right] x
\end{aligned}
$$

where the time constants are

$$
T_{i}=\frac{A_{i}}{a_{i}} \sqrt{\frac{2 h_{i}^{0}}{g}}, \quad i=1, \cdots, 4 .
$$

The corresponding transfer matrix is

$$
G(s)=\left[\begin{array}{cc}
\frac{\gamma_{1} c_{1}}{1+s T_{1}} & \frac{\left(1-\gamma_{2}\right) c_{1}}{\left(1+s T_{3}\right)\left(1+s T_{1}\right)} \\
\frac{\left(1-\gamma_{1}\right) c_{2}}{\left(1+s T_{4}\right)\left(1+s T_{2}\right)} & \frac{\gamma_{2} c_{2}}{1+s T_{2}}
\end{array}\right]
$$

where $c_{1}=T_{1} k_{1} k_{c} / A_{1}$ and $c_{2}=T_{2} k_{2} k_{c} / A_{2}$. Note the way $\gamma_{1}$ and $\gamma_{2}$ enter the matrix.
For the two operating points $P_{-}$and $P_{+}$we have the following time constants:

$$
\begin{array}{ccc} 
& P_{-} & P_{+} \\
\hline\left(T_{1}, T_{2}\right) & (62,90) & (63,91) \\
\left(T_{3}, T_{4}\right) & (23,30) & (39,56)
\end{array} .
$$

The dominating time constants are thus similar for both operating conditions. The physical modeling gives the two transfer matrices

$$
G_{-}(s)=\left[\begin{array}{cc}
\frac{2.6}{1+62 s} & \frac{1.5}{(1+23 s)(1+62 s)} \\
\frac{1.4}{(1+30 s)(1+90 s)} & \frac{2.8}{1+90 s}
\end{array}\right]
$$

and

$$
G_{+}(s)=\left[\begin{array}{cc}
\frac{1.5}{1+63 s} & \frac{2.5}{(1+39 s)(1+63 s)} \\
\frac{2.5}{(1+56 s)(1+91 s)} & \frac{1.6}{1+91 s}
\end{array}\right] .
$$

Figs. 3 and 4 show simulations of these two models compared to real data obtained from the identification experiments discussed in Section V. The inputs are pseudorandom binary sequences (PRBS's) with low amplitudes, so that the dynamics are captured by a linear model. The model outputs agree well with the responses of the real process.

The quadruple-tank process is simple to build. The four tanks are made out of plexiglas tubes. The height of each tank is $20 \mathrm{~cm}$ and the diameter is about $6 \mathrm{~cm}$. The pumps are gear pumps with a capacity of $2.5 \ell / \mathrm{min}$. Capacitive electrodes are used for measuring the water levels. The tanks and the pumps are connected by flexible tubings, each with a diameter of $6 \mathrm{~mm}$. The equipment is of the same type as used for the double tank process described in [5] and [4].

\section{INTERPRETATION OF MULTIVARIABLE Zero}

The zero locations and their directions of the transfer matrix $G$ are derived in this section. It is shown that they have intuitive physical interpretations in terms of how the valves $\gamma_{1}$ and $\gamma_{2}$ are 

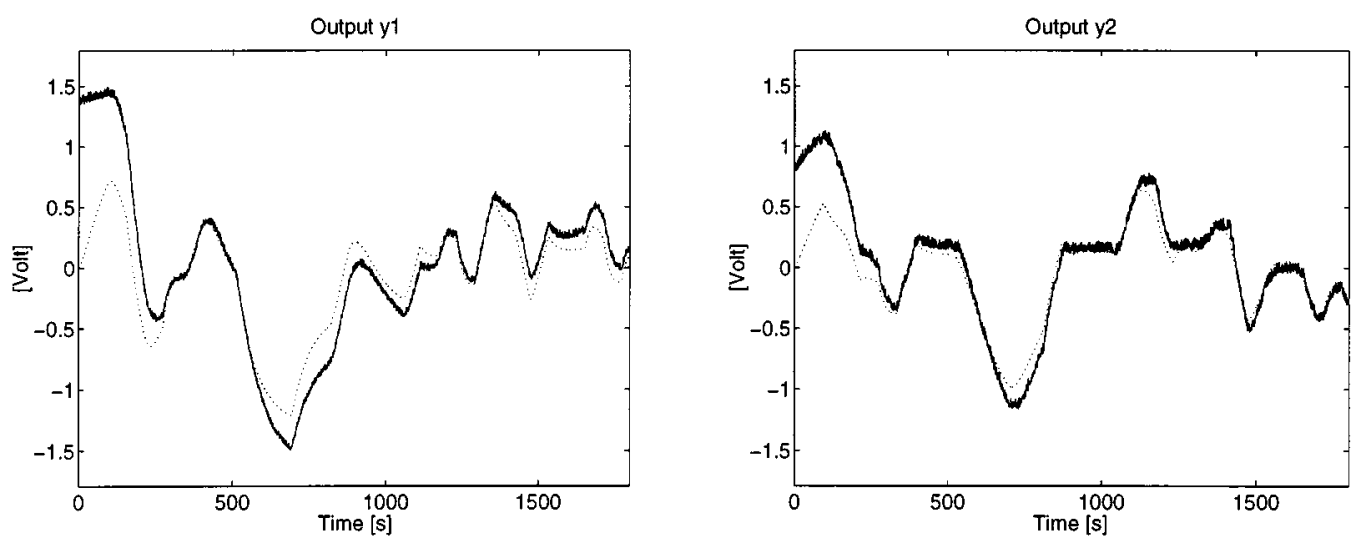

Fig. 4. Validation of the nonminimum-phase model $G_{+}$. Same variables as in Fig. 3 are shown.

set. A simple expression for the relative gain array of the model is also derived.

\section{A. Zero Location}

The zeros of the transfer matrix in (4) are the zeros of the numerator polynomial of the rational function

$$
\begin{aligned}
\operatorname{det} G(s)= & \frac{c_{1} c_{2}}{\gamma_{1} \gamma_{2} \prod_{i=1}^{4}\left(1+s T_{i}\right)} \\
& \times\left[\left(1+s T_{3}\right)\left(1+s T_{4}\right)-\frac{\left(1-\gamma_{1}\right)\left(1-\gamma_{2}\right)}{\gamma_{1} \gamma_{2}}\right] .
\end{aligned}
$$

The transfer matrix $G$ thus has two finite zeros for $\gamma_{1}, \gamma_{2} \in$ $(0,1)$. One of them is always in the left half-plane, but the other can be located either in the left or the right half-plane, as follows from the following root-locus argument. Introduce a parameter $\eta \in(0, \infty)$ as

$$
\eta:=\left(1-\gamma_{1}\right)\left(1-\gamma_{2}\right) / \gamma_{1} \gamma_{2}
$$

If $\eta$ is small, the two zeros are close to $-1 / T_{3}$ and $-1 / T_{4}$, respectively. Furthermore, one zero tends to $-\infty$ and one zero tends to $+\infty$ as $\eta \rightarrow \infty$. If $\eta=1$ one zero is located at the origin. This case corresponds to $\gamma_{1}+\gamma_{2}=1$. It follows that the system is nonminimum phase for

$$
0<\gamma_{1}+\gamma_{2}<1
$$

and minimum phase for

$$
1<\gamma_{1}+\gamma_{2}<2
$$

Recall that $\gamma_{1}+\gamma_{2}=1.30>1$ for $P_{-}$and $\gamma_{1}+\gamma_{2}=0.77<1$ for $P_{+}$. Fig. 5 shows the location of the two operating points $P_{-}$ and $P_{+}$. For operating points above the solid line the system is minimum phase and below it is nonminimum phase.

The multivariable zero being in the left or in right half-plane has a straightforward physical interpretation. Let $q_{i}$ denote the flow through Pump $i$ and assume that $q_{1}=q_{2}$. Then the sum of the flows to the upper tanks is $\left[2-\left(\gamma_{1}+\gamma_{2}\right)\right] q_{1}$ and the sum of the flows to the lower tanks is $\left(\gamma_{1}+\gamma_{2}\right) q_{1}$. Hence, the flow

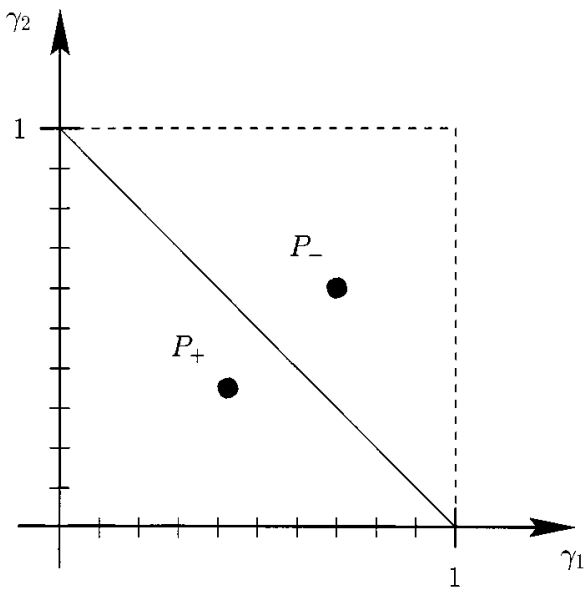

Fig. 5. Depending on the values of the valve parameters $\gamma_{1}$ and $\gamma_{2}$ the system is minimum or nonminimum phase. Above the straight line between $(0,1)$ and $(1,0)$ the system is minimum phase and below it is nonminimum phase. The two cases studied in this paper corresponds to the two dots.

to the lower tanks is greater than the flow to the upper tanks if $\gamma_{1}+\gamma_{2}>1$, i.e., if the system is minimum phase. The flow to the lower tanks is smaller than the flow to the upper tanks if the system is nonminimum phase. It is intuitively easier to control $y_{1}$ with $u_{1}$ and $y_{2}$ with $u_{2}$, if most of the flows goes directly to the lower tanks. The control problem is particularly hard if the total flow going to the left tanks (Tanks 1 and 3) is equal to the total flow going to the right tanks (Tanks 2 and 4). This corresponds to $\gamma_{1}+\gamma_{2}=1$, i.e., a multivariable zero in the origin. There is thus an immediate connection between the zero location of the model and physical intuition of controlling the quadruple-tank process.

\section{B. Zero Direction}

An important difference between scalar systems and multivariable systems is that not only the location of a multivariable zero is important but also its direction. We define the (output) direction of a zero $z$ as a vector $\psi \in \mathbb{R}^{2}$ of unit length such as $\psi^{T} G(z)=0$. If $\psi$ is parallel to a unit vector, then the zero is only associated with one output. If this is not the case, then the effect of a right half-plane zero may be distributed between both 
outputs. See [30] for further discussions. For the transfer matrix $G$ in (4), the zero direction for $z>0$ is given by

$$
\left[\begin{array}{l}
\psi_{1} \\
\psi_{2}
\end{array}\right]^{T}\left[\begin{array}{cc}
\frac{\gamma_{1} c_{1}}{1+z T_{1}} & \frac{\left(1-\gamma_{2}\right) c_{1}}{\left(1+z T_{3}\right)\left(1+z T_{1}\right)} \\
\frac{\left(1-\gamma_{1}\right) c_{2}}{\left(1+z T_{4}\right)\left(1+z T_{2}\right)} & \frac{\gamma_{2} c_{2}}{1+z T_{2}}
\end{array}\right]=\left[\begin{array}{l}
0 \\
0
\end{array}\right]^{T} .
$$

Note that it follows from this equation that $\psi_{1}, \psi_{2} \neq 0$, so the zero is never associated with only one output. If we solve (8) for $\gamma_{2}$ and simplify, it is easy to show that

$$
\frac{\psi_{1}}{\psi_{2}}=-\frac{1-\gamma_{1}}{\gamma_{1}} \cdot \frac{c_{2}\left(1+z T_{1}\right)}{c_{1}\left(1+z T_{4}\right)\left(1+z T_{2}\right)} .
$$

From this equation it is possible to conclude that if $\gamma_{1}$ is small, then $z$ is mostly associated with the first output. If $\gamma_{1}$ is close to one, then $z$ is mostly associated with the second output. Hence, for a given zero location, the relative size of $\gamma_{1}$ and $\gamma_{2}$ determines which output the right half-plane zero is related to.

\section{Relative Gain Array}

The relative gain array (RGA) was introduced by Bristol [7] as a measure of interaction in multivariable control systems. The RGA $\Lambda$ is defined as $\Lambda=G(0) * G^{-T}(0)$, where the asterisk denotes the Schur product (element-by-element matrix multiplication) and $-T$ inverse transpose. It is possible to show that the elements of each row and column of $\Lambda$ sum up to one, so for a $2 \times 2$ system the RGA is determined by the scalar $\lambda=\Lambda_{11}$. The RGA is used as a tool mainly in the process industry to decide on control structure issues such as input-output pairing for decentralized controllers [27]. See [13] for a summary of closed-loop properties predicted from the RGA. McAvoy [25] proposed that one should strive for a pairing with $0.67<\lambda<1.50$. The system is particularly hard to control if $\lambda<0$.

The RGA of the quadruple-tank process is given by the simple expression

$$
\lambda=\frac{\gamma_{1} \gamma_{2}}{\gamma_{1}+\gamma_{2}-1}
$$

Note that the RGA is only depending on the valve settings and no other physical parameters of the process. Fig. 6 shows a contour plot of $\lambda$ as a function of $\gamma_{1}$ and $\gamma_{2}$. The edges of the box $0 \leq \gamma_{1}, \gamma_{2} \leq 1$ corresponds to $\lambda=0$ and $\lambda=1$ as is shown in the figure. The magnitude of $\lambda$ increases as $\gamma_{1}+\gamma_{2}$ becomes close to one. There are no $\gamma_{1}, \gamma_{2} \in(0,1)$ such that $\lambda \in(0,1)$. From (10) we see for instance that $\lambda<0$ if $\gamma_{1}+\gamma_{2}<1$, which corresponds to the nonminimum-phase setting discussed previously.

If the valves of the quadruple-tank process are set such that $\gamma_{1}+\gamma_{2}<1$, then the RGA analysis suggests that another input-output pairing for decentralized control should be chosen. Let

$$
\widetilde{G}=\left[\begin{array}{ll}
G_{21} & G_{22} \\
G_{11} & G_{12}
\end{array}\right]
$$

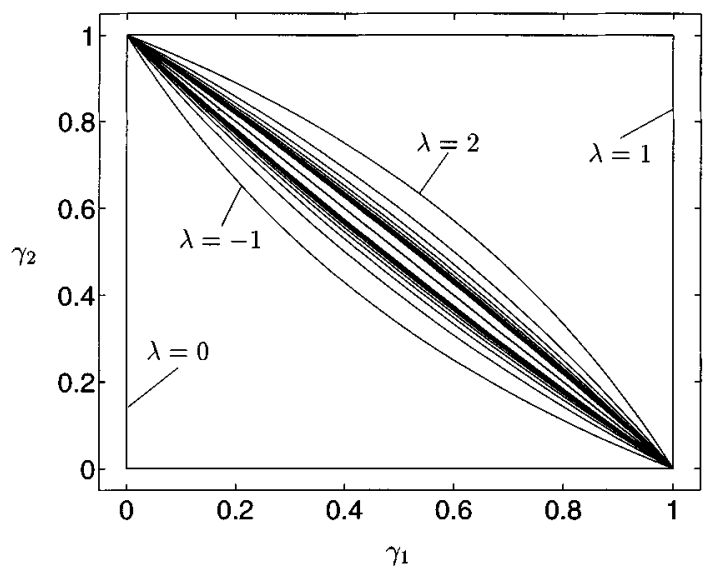

Fig. 6. Contour plot of the RGA $\lambda$ as a function of the valve parameters $\gamma_{1}$ and $\gamma_{2}$. The plot is drawn for $\lambda=-10,-9, \ldots, 10$. We have $\lambda \leq 0$ below the straight line between $(0,1)$ and $(1,0)$ and $\lambda \geq 1$ above the line. Close to the line, $|\lambda|$ is large.

be the linearized model with $y_{1}$ and $y_{2}$ permuted. The RGA for $\widetilde{G}$ is

$$
\widetilde{\lambda}=\frac{\left(1-\gamma_{1}\right)\left(1-\gamma_{2}\right)}{1-\gamma_{1}-\gamma_{2}} .
$$

Hence, if $\gamma_{1}+\gamma_{2}<1$ then $\widetilde{\lambda}>0$ so a decentralized control structure corresponding to $\widetilde{G}$ is preferable according to the RGA. This is intuitive from physical considerations.

\section{Data for the Laboratory Process}

We sum up the physical interpretations of the zero as well as of the RGA and give the corresponding values for the real process. One zero of $G$ is always in the left half-plane. The other can be located anywhere on the real axis. The location is determined by how the valves corresponding to the parameters $\gamma_{1}$ and $\gamma_{2}$ are adjusted. If $\gamma_{1}+\gamma_{2} \in(0,1)$ then the zero is in the right half-plane, while if $\gamma_{1}+\gamma_{2} \in(1,2)$ the zero is in the left half-plane. The relative size of $\gamma_{1}$ and $\gamma_{2}$ gives the zero direction, i.e., $\gamma_{1} / \gamma_{2}$ tells which output the effect of the zero is associated to. If $\gamma_{1} / \gamma_{2}$ is small, then the zero is associated with output one and vice versa. The RGA of the quadruple-tank process is given by $\gamma_{1} \gamma_{2} /\left(\gamma_{1}+\gamma_{2}-1\right)$ and is thus only dependent on the valve settings.

For the two operating points $P_{-}$and $P_{+}$we have the following zeros:

$$
\begin{array}{ccc} 
& P_{-} & P_{+} \\
\hline \text { Zeros } & (-0.060,-0.018) & (-0.057,0.013)
\end{array}
$$

Hence, $G_{+}$has a zero in the right half-plane, which (as we will see in Section VI) deteriorate the performance considerably. The direction of the zero $z=0.013$ is given by $\psi_{1} / \psi_{2}=-0.85$. The RGA for $P_{-}$is given by $\lambda=1.40$ and for $P_{+}$by $\lambda=-0.64$. RGA analysis indicates that the nonminimum-phase system is harder to control than the minimum-phase system. For the system $\widetilde{G}(G$ with permuted outputs) the RGA is $\widetilde{\lambda}=-0.40$ for the minimum-phase setting and $\widetilde{\lambda}=1.64$ for the nonminimum-phase setting. There 
exist results that connect the RGA and the zero location, for example, see [32].

\section{PROPERTIES OF NONLINEAR MODEL}

The characteristics of the nonlinear model (1) of the quadruple-tank process is studied in this section. The nonlinear zero dynamics are derived and features of the stationary operating points are given.

\section{A. Nonlinear Zero Dynamics}

It is possible to choose an input $\left(v_{1}, v_{2}\right)$ and an initial state $\left(h_{1}^{0}, \cdots, h_{4}^{0}\right)$ for the system (1) such that $h_{1}(t)=h_{2}(t)=0$ for all $t \geq 0$. The constrained dynamics are called the zero dynamics [14]. The zero dynamics of (1) are

$$
\begin{aligned}
\frac{d h_{3}}{d t} & =-\frac{a_{3}}{A_{3}} \sqrt{2 g h_{3}}-\frac{\left(1-\gamma_{2}\right) a_{4}}{\gamma_{2} A_{3}} \sqrt{2 g h_{4}} \\
\frac{d h_{4}}{d t} & =-\frac{\left(1-\gamma_{1}\right) a_{3}}{\gamma_{1} A_{4}} \sqrt{2 g h_{3}}-\frac{a_{4}}{A_{4}} \sqrt{2 g h_{4}}
\end{aligned}
$$

The characteristic equation for the linearized dynamics of (11) is

$$
\left(1+s T_{3}\right)\left(1+s T_{4}\right)-\frac{\left(1-\gamma_{1}\right)\left(1-\gamma_{2}\right)}{\gamma_{1} \gamma_{2}}=0
$$

where $T_{3}$ and $T_{4}$ are the time constants defined in (3). The solutions of this equation are equal to the zeros of $G$, compare (7). In particular, it follows that the nonlinear system is nonminimum phase for $0<\gamma_{1}+\gamma_{2}<1$ and minimum phase for $1<\gamma_{1}+\gamma_{2}<2$. This is not surprising since the operations of taking a linear approximation and calculating the zero dynamics commute [14].

Some nonlinear design methods like feedback linearization techniques require that the zero dynamics are stable. Modifications to these have been proposed to extend the applicability to nonminimum-phase systems. The nonlinear zero dynamics of the quadruple-tank process can be made stable or unstable by simply changing a valve. The process is thus suitable to illustrate how sensitive a nonlinear design method is to the stability of the zero dynamics.

\section{B. Steady-State Conditions}

For a stationary operating point $\left(h^{0}, v^{0}\right)$, the differential equations in (1) give that

$$
\begin{aligned}
& \frac{a_{3}}{A_{3}} \sqrt{2 g h_{3}^{0}}=\frac{\left(1-\gamma_{2}\right) k_{2}}{A_{3}} v_{2}^{0} \\
& \frac{a_{4}}{A_{4}} \sqrt{2 g h_{4}^{0}}=\frac{\left(1-\gamma_{1}\right) k_{1}}{A_{4}} v_{1}^{0}
\end{aligned}
$$

and thus

$$
\begin{aligned}
& \frac{a_{1}}{A_{1}} \sqrt{2 g h_{1}^{0}}=\frac{\gamma_{1} k_{1}}{A_{1}} v_{1}^{0}+\frac{\left(1-\gamma_{2}\right) k_{2}}{A_{1}} v_{2}^{0} \\
& \frac{a_{2}}{A_{2}} \sqrt{2 g h_{2}^{0}}=\frac{\left(1-\gamma_{1}\right) k_{1}}{A_{2}} v_{1}^{0}+\frac{\gamma_{2} k_{2}}{A_{2}} v_{2}^{0} .
\end{aligned}
$$

It follows that there exists a unique constant input $\left(v_{1}^{0}, v_{2}^{0}\right)$ giving the steady-state levels $\left(h_{1}^{0}, h_{2}^{0}\right)$ if and only if the matrix

$$
\left[\begin{array}{cc}
\gamma_{1} k_{1} & \left(1-\gamma_{2}\right) k_{2} \\
\left(1-\gamma_{1}\right) k_{1} & \gamma_{2} k_{2}
\end{array}\right]
$$

is nonsingular, i.e., if and only if $\gamma_{1}+\gamma_{2} \neq 1$. The singularity of this matrix is natural. In stationarity, the flow through Tank 1 is $\gamma_{1} q_{1}+\left(1-\gamma_{2}\right) q_{2}$ and the flow through Tank 2 is $\gamma_{2} q_{2}+(1-$ $\left.\gamma_{1}\right) q_{1}$, where $q_{i}$ is the flow through Pump $i$. If $\gamma_{1}+\gamma_{2}=1$, these flows equal $\gamma_{1}\left(q_{1}+q_{2}\right)$ and $\left(1-\gamma_{1}\right)\left(q_{1}+q_{2}\right)$, respectively. The stationary flows through Tanks 1 and 2 are thus dependent, and so must the levels also be. Note that the condition $\gamma_{1}+\gamma_{2}=1$ is equivalent to that $G$ in (4) has a zero in the origin.

\section{SYSTEM IDENTIFICATION}

Estimation of models based on experimental data is described next. Standard system identification techniques are used [23]. It is shown that the identified models are accurate and agree well with the physical model described previously.

Both single input-multiple output (SIMO) and multiple input-multiple output (MIMO) identification experiments were performed with PRBS signals as inputs. The levels of the PRBS signals were chosen so that the process dynamics were approximately linear.

Black-box and gray-box identification methods were tested using Matlab's System Identification Toolbox [22]. Linear single input-single output (SISO), multiple input-single output (MISO), and MIMO models were identified in ARX, ARMAX, and state-space forms. All model structures gave similar responses to validation data. Here we only present some examples of the results. We start with a black-box approach. Fig. 7 shows validation data for the minimum-phase setting together with a simulation of a state-space model derived with the subspace algorithm N4SID [22], [34]. The state-space model has three real poles corresponding to time constants 8,41 , and 113. It has one multivariable zero in -0.99 . The simulation of the nonminimum-phase model is shown in Fig. 8. This model is of fourth order and has time constants 11, 31, 140, and 220. Its two zeros are located in -0.288 and 0.019 . The validation results are of similar quality as the result for the physical models shown in Figs. 3 and 4. Note that the minimum-phase setting gives an identified model with no right half-plane zero, whereas the nonminimum-phase setting gives a dominating right half-plane zero, i.e., a right half-plane zero close to the origin compared to the time scale given by the time constants. This agrees with the physical model.

Gray-box models with structure fixed to the linear state-space equation (2) gave similar validation results as the ones previously shown. Because of the fixed structure, the number of poles and zeros are the same as for the physical model. For the minimum-phase setting we have time constants $\left(T_{1}, T_{2}, T_{3}, T_{4}\right)=$ $(96,99,32,39)$ and zeros at -0.045 and -0.012 , whereas for the nonminimum-phase setting we have $\left(T_{1}, T_{2}, T_{3}, T_{4}\right)=$ $(77,112,53,55)$ and zeros at 0.014 and -0.051 . The zeros agree very well with the ones derived from the physical model. 

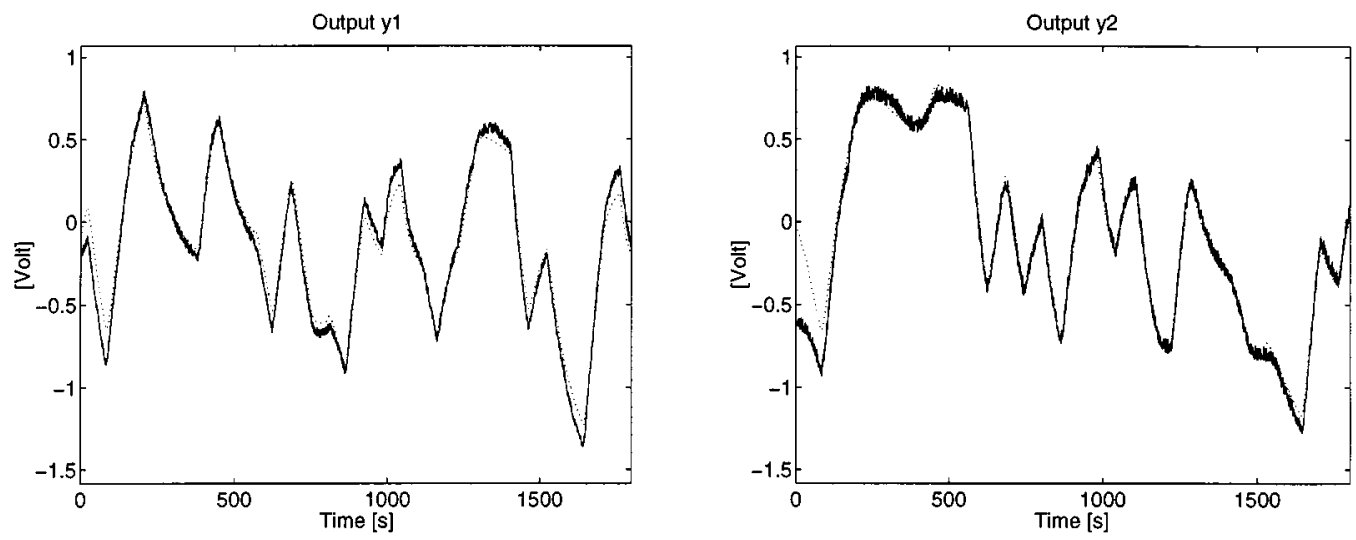

Fig. 7. Validation of identified state-space model for the minimum-phase setting. Outputs from model (dashed) together with the outputs from the real process (solid) are shown.
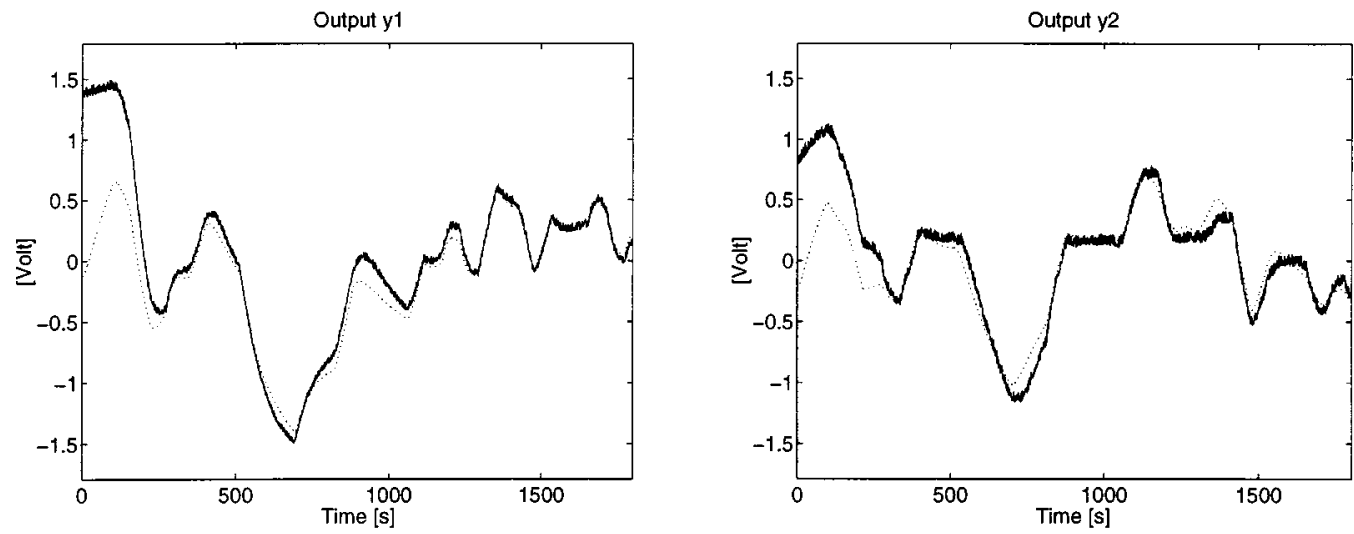

Fig. 8. Validation of identified state-space model for the nonminimum-phase setting. Same variables as in Fig. 7 are shown.

The conclusion of this section is that the quadruple-tank process is also suitable to illustrate multivariable system identification techniques.

\section{DeCENTRALIZED PI CONTROL}

The decentralized control law $u=\operatorname{diag}\left\{C_{1}, C_{2}\right\}(r-y)$ in Fig. 9 is in this section applied to the real process as well as to nonlinear and linear process models. PI controllers of the form

$$
C_{\ell}(s)=K_{\ell}\left(1+\frac{1}{T_{i \ell} s}\right), \quad \ell=1,2
$$

are tuned manually based on simulations of the linear physical models (5) and (6).

For the minimum-phase setting it is easy to find controller parameters that give good performance. The controller settings $\left(K_{1}, T_{i 1}\right)=(3.0,30)$ and $\left(K_{2}, T_{i 2}\right)=(2.7,40)$ give the responses shown in Fig. 10. The responses are given for a step in the reference signal $r_{1}$. The top four plots show control of the simulated nonlinear model in (1) (dashed lines) and control of an identified linear state-space model (solid). The four lower plots show the responses of the real process. The discrepancies between simulations and the true time responses are small.

Manually tuning to find controller parameters that give good closed-loop performance is difficult for the nonminimum-phase setting. This is, of course, due to that the nonminimum-phase

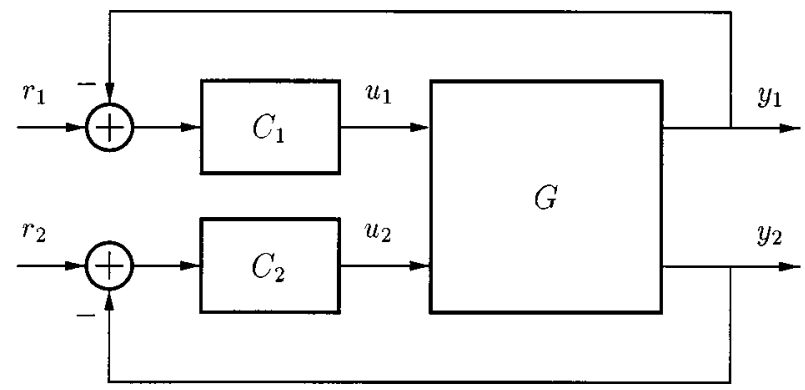

Fig. 9. Decentralized control structure with two PI controllers $C_{1}$ and $C_{2}$.

process is generically harder to control than the minimum-phase process. The controller parameters $\left(K_{1}, T_{i 1}\right)=(1.5,110)$ and $\left(K_{2}, T_{i 2}\right)=(-0.12,220)$ stabilize the process and give reasonable performance, see Fig. 11. They give, however, much slower responses than in the minimum-phase case. Note the different time scales compared to Fig. 10. The settling time is approximately ten times longer for the nonminimum-phase setting. (The control signal $u_{2}$ seems to be noiseless in Fig. 11, which is due to the low gain $K_{2}$.) Note that the closed-loop performance will be better if $y_{1}$ and $y_{2}$ are permuted in the control structure in Fig. 9, as was suggested by the RGA analysis in Section III. Recall that the RGA is given by $\lambda=-0.64$ for the original pairing and $\widetilde{\lambda}=1.64$ if the process outputs are permuted. The permutation, however, does not change the location 

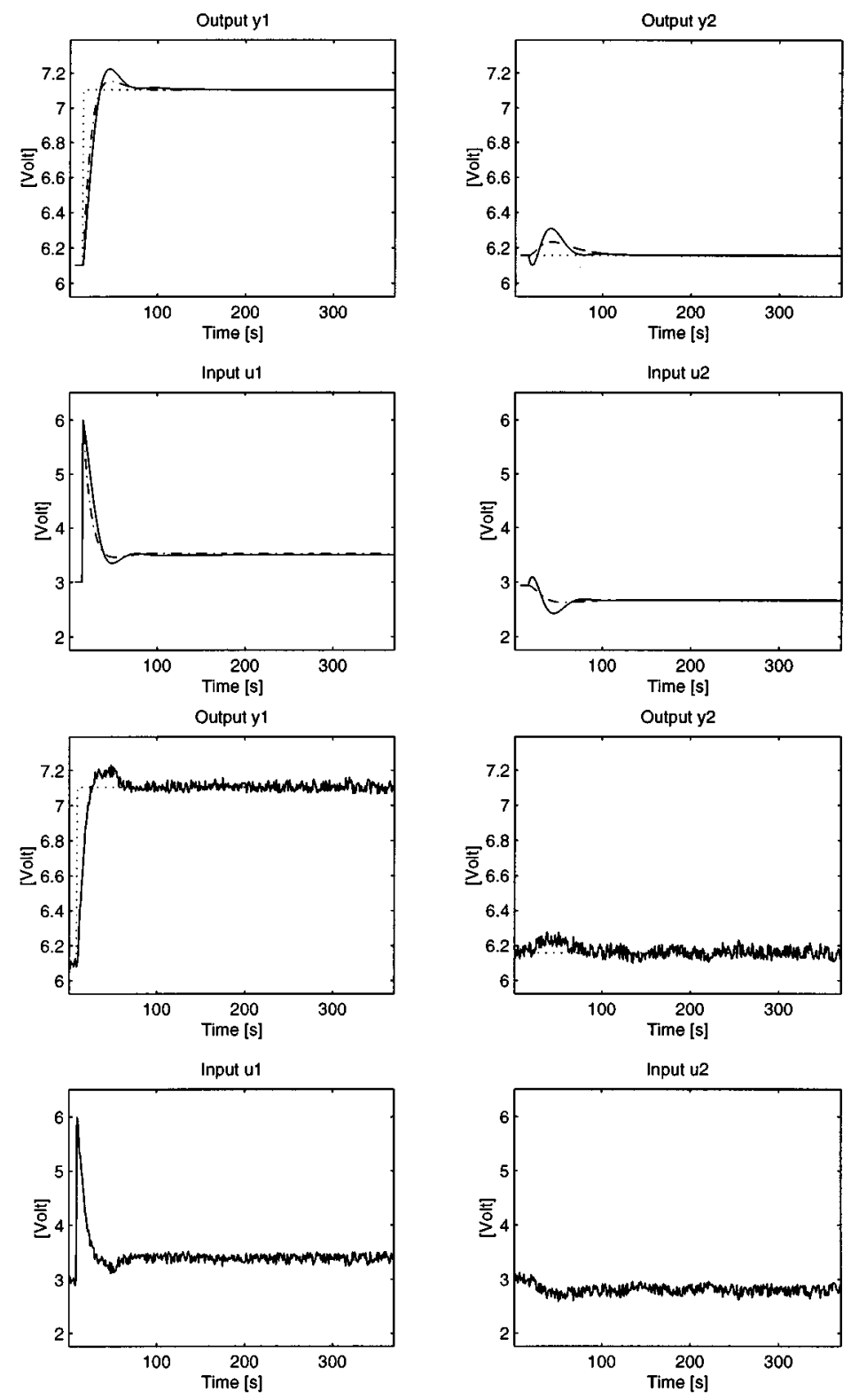

Fig. 10. Results of PI control of minimum-phase system. The upper four plots show simulations with the nonlinear physical model (dashed) and the identified linear model (solid). The four lower plots show experimental results.

of the right half-plane zero, and experiments have shown that the settling times are still much larger than for the minimum-phase setting [29].

Centralized multivariable control has also been tested on the quadruple-tank process [12]. These experiments indicate that for the minimum-phase system it is not possible to achieve much faster response than with the decentralized PI controller in this section. For the nonminimum-phase case, however, a multivariable controller based on $H_{\infty}$ design methods gave $30-40 \%$ faster settling time than the responses shown here. Note that this is still several times slower than the response time of the minimum-phase system. An interesting property of the $H_{\infty}$ controller for the nonminimum-phase system is that it happens to get an dominating antidiagonal structure, contrary to the diagonal controller described in this section. This is intuitive and agrees also with the RGA analysis, which suggested a permutation of $y_{1}$ and $y_{2}$. Multivariable controller-tuning method based on relay feedback experiments have also been investigated on the quadruple-tank process [17], [29]. It was shown in [29] that several of the methods proposed in the literature cannot handle automatic control design for both the minimum-phase and the nonminimum-phase setting.

The minimal achievable settling times for the step responses are important characteristics of the system. A lower bound on the settling time for multivariable control systems is derived in [16] by generalizing scalar results by Middleton [26]. It is shown that given a fixed undershoot in $y_{1}$ and a fixed interaction to $y_{2}$, then, if the right half-plane zero is close to the origin, the step response will necessarily have a large settling time with any linear controller. The bound also takes the direction of the zero into account. Using the lower bound in [16], it is possible to show that the nonminimum-phase system must have a settling time that 

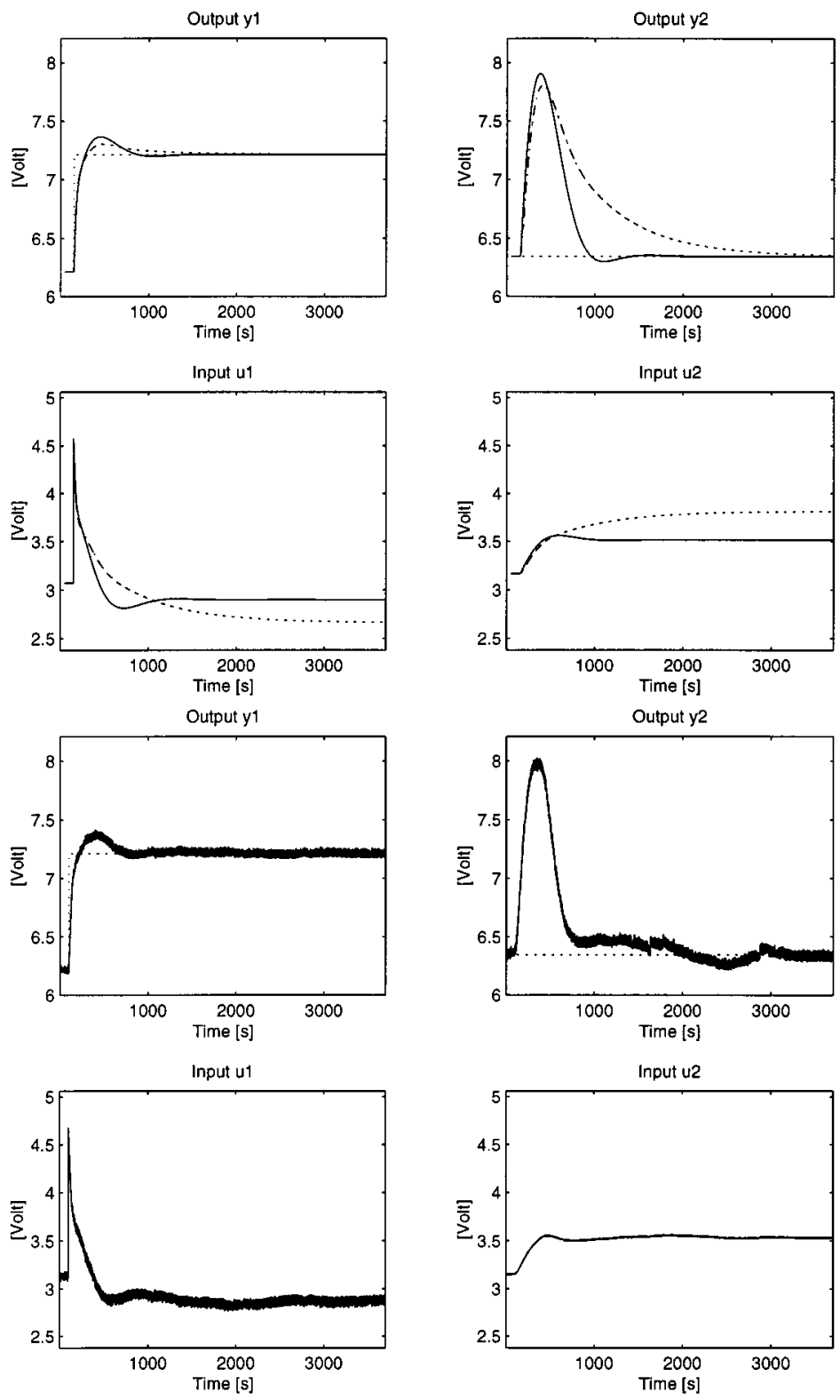

Fig. 11. Results of PI control of nonminimum-phase system. Same variables are shown as in Fig. 10. Note the ten times longer time scales.

is considerably larger than the minimum-phase response shown in this paper. The minimum-phase setting of the quadruple-tank process can theoretically be arbitrarily tight controlled with a decentralized controller [15].

\section{CONCLUSIONS}

A new multivariable laboratory process that consists of four interconnected water tanks has been described. A motivation for developing this process was to illustrate concepts in multivariable control or as stated in [21]:

[The control laboratory's] main purpose is to provide the connection between abstract control theory and the real world. Therefore it should give an indication of how control theory can be applied and also an indication of some of its limitations.
It was shown that the quadruple-tank process is well suited to illustrate performance limitations in multivariable control design caused by right half-plane zeros. This followed from that the linearized model of the process has a multivariable zero that in a direct way is connected to the physical positions of two valves. The positions are given by two parameters $\gamma_{1}, \gamma_{2} \in(0,1)$. It was shown that $\gamma_{1}+\gamma_{2}$ determines the location of the zero, so that if $\gamma_{1}+\gamma_{2}<1$ the system is nonminimum phase and if $\gamma_{1}+\gamma_{2}>1$ the system is minimum phase. The quotient $\gamma_{1} / \gamma_{2}$ gives the zero direction.

Models from physical data and experimental data were derived and they were shown to have responses similar to the real process. Decentralized PI control showed that it was much more difficult to control the process in the nonminimum-phase setting than in the minimum-phase setting.

The quadruple-tank process is used today in several courses in the control education at LTH, Lund, Sweden, and at KTH, 
Stockholm, Sweden. The experiments described in this paper have been performed using the PC interface shown in Fig. 1 [28] which has been developed in the man-machine interface generator InTouch from Wonderware Corporation. The interface is connected to the real process as well as to a real-time kernel [2]. This gives a flexible experimental platform where controllers can be designed in Matlab, loaded into the interface, simulated with the nonlinear model, and finally tested on the real process.

\section{ACKNOWLEDGMENT}

The author would like to thank J. L. Rocha Nunes for doing the experiments shown in this paper, when he was visiting Lund Institute of Technology as an ERASMUS student. The laboratory process was built by R. Braun. The author would also like to thank K. J. Åström and P. Hagander for their comments on the manuscript.

\section{REFERENCES}

[1] M. Åkesson, E. Gustafson, and K. H. Johansson, "Control design for a helicopter lab process," in IFAC'96, Preprints 13th World Congr. IFAC, San Francisco, CA, July 1996.

[2] L. Andersson and A. Blomdell, "A real-time programming environment and a real-time kernel," in Nat. Swedish Symp. Real-Time Syst., L. Asplund, Ed. Uppsala, Sweden: Dept. Comput. Syst., Uppsala Univ., 1991.

[3] K. J. Åström, "Fundamental limitations of control system performance," in Mathematical Engineering: A Kailath Festschrift, A. Paulraj, C. Schaper, and V. Roychowdhury, Eds. Boston, MA: Kluwer, 1996.

[4] K. J. Åström and M. Lundh, "Lund control program combines theory with hands-on experience," IEEE Contr. Syst. Mag., vol. 12, no. 3, pp. 22-30, 1992.

[5] K. J. Åström and A.-B. Östberg, "A teaching laboratory for process control,” IEEE Contr. Syst. Mag., vol. 6, pp. 37-42, 1986.

[6] H. W. Bode, Network Analysis and Feedback Amplifier Design. New York: Van Nostrand, 1945.

[7] E. Bristol, "On a new measure of interaction for multivariable process control," IEEE Trans. Automat. Contr., vol. 11, p. 133, 1966.

[8] E. J. Davison, "Description of multivariable apparatus for real time-control studies (MARTS),” Dept. Elect. Eng., Univ. Toronto, Canada, Tech. Rep. 8514a, 1985 .

[9] B. A. Francis, A Course in $H_{\infty}$ Control Theory. Berlin, Germany: Springer-Verlag, 1987.

[10] J. Freudenberg and D. Looze, Frequency Domain Properties of Scalar and Multivariable Feedback Systems. Berlin, Germany: Springer-Verlag, 1988.

[11] G. C. Goodwin, "Defining the performance envelope in industrial control," in 16th Amer. Contr. Conf., Albuquerque, NM, 1997, Plenary Session 1 .

[12] M. Grebeck, "A comparison of controllers for the quadruple tank system," Department of Automatic Control, Lund Institute of Technology, Lund, Sweden, Tech. Rep., 1998.

[13] P. Grosdidier, M. Morari, and B. R. Holt, "Closed-loop properties from steady-state gain information," Ind. Eng. Chem. Fundam., vol. 24, no. 2, pp. 221-235, 1985 .

[14] A. Isidori, Nonlinear Control Systems, 2nd ed: Springer-Verlag, 1989.

[15] K. H. Johansson, "Relay feedback and multivariable control," Ph.D. dissertation, Dept. Automat. Contr., Lund Inst. Technol., Lund, Sweden, Nov. 1997.

[16] — " "Interaction bounds in multivariable control systems," in IFAC World Congr., Beijing, China, 1999.

[17] K. H. Johansson, B. James, G. F. Bryant, and K. J. Åström, "Multivariable controller tuning," in 17th Amer. Contr. Conf., Philadelphia, PA 1998.
[18] K. H. Johansson and J. L. R. Nunes, "A multivariable laboratory process with an adjustable zero," in 17th Amer. Contr. Conf., Philadelphia, PA, 1998.

[19] K. H. Johansson and A. Rantzer, "Multiloop control of minimum phase processes," in Proc. 16th Amer. Contr. Conf., Albuquerque, NM, 1997.

[20] — "Decentralized control of sequentially minimum phase systems," IEEE Trans. Automat. Contr., vol. 44, pp. 1909-1913, 1999.

[21] N. A. Kheir, K. J. Åström, D. Austander, K. C. Cheok, G. F. Franklin, M. Masten, and M. Rabins, "Control systems engineering education," Automatica, vol. 32, no. 2, pp. 147-166, 1996.

[22] L. Ljung, System Identification Toolbox, Version 4.0.3, The Mathworks, Inc, 1997.

[23] — System Identification - Theory for the User, 2nd ed. Englewood Cliffs, NJ: Prentice-Hall, 1999.

[24] M. Mansour and W. Schaufelberger, "Software and laboratory experiment using computers in control education," IEEE Contr. Syst. Mag., vol. 9, no. 3, pp. 19-24, 1989.

[25] T. J. McAvoy, "Interaction analysis: Principles and applications," Instrument Soc. Amer., Research Triangle Park, NC, 1983.

[26] R. H. Middleton, "Trade-offs in linear control system design," Auto matica, vol. 27, no. 2, pp. 281-292, 1991.

[27] M. Morari and E. Zafiriou, Robust Process Control. Englewood Cliffs, NJ: Prentice-Hall, 1989.

[28] J. R. Nunes, "Modeling and control of the quadruple-tank process," Master thesis, Dept. Automat. Contr., Lund Inst. Technol., Lund, Sweden, 1997.

[29] V. Recica, "Automatic tuning of multivariable controllers," Master thesis, Dept. Automat. Contr., Lund Inst. Technol., Lund, Sweden, 1998.

[30] M. M. Seron, J. H. Braslavsky, and G. C. Goodwin, Fundamental Limitations in Filtering and Control. New York: Springer-Verlag, 1997.

[31] F. G. Shinskey, "Controlling Multivariable Processes," Instrument Society of America, Research Triangle Park, NC, 1981.

[32] S. Skogestad and I. Postlethwaite, Multivariable Feedback Control-Analysis and Design. New York: Wiley, 1996.

[33] G. Stein, "Respect the unstable," in 30th IEEE Conf. Decision Contr. Honolulu, HI, 1990

[34] P. Van Overschee and B. De Moor, "N4SID: Subspace algorithms for the identification of combined deterministic-stochastic systems," Automatica, vol. 30, no. 1, pp. 75-93, 1994.

[35] D. Vischer and H. Bleuler, "A new approach to sensorless and voltage controlled AMB's based on network theory concepts," in 2nd Int. Symp. Magnetic Bearings, 1990.

[36] G. Zames, "Feedback and optimal sensitivity: Model reference transformations, multiplicative seminorms and approximate inverses," IEEE Trans. Automat. Contr., vol. AC-26, pp. 301-320, 1981.

[37] G. Zames and D. Bensoussan, "Multivariable feedback, sensitivity, and decentralized control," IEEE Trans. Automat. Contr., vol. AC-28, pp. 1030-1035, 1983

[38] G. Zames and B. A. Francis, "Feedback, minimax sensitivity, and optimal robustness," IEEE Trans. Automat. Contr., vol. AC-28, pp. 585-601, 1983.

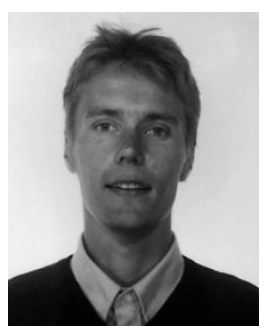

Karl Henrik Johansson (S'92-M'98) received the M.S. degree in electrical engineering in 1992 and the $\mathrm{Ph} . \mathrm{D}$. degree in control engineering in 1997, both from Lund University, Sweden.

He currently holds a postdoctoral position at the Department of Electrical Engineering and Computer Sciences, University of California, Berkeley. His research interests are in hybrid systems, multivariable control, relay controller turning, and various applications of control. He also has a great interest in control education.

Dr. Johansson was awarded the Young Author Prize at the IFAC World Congress 1996 (together with Dr. A. Rantzer). He received the Peccei Award 1993 from the International Institute of Applied Systems Analysis in Austria and the Scania Research Award 1996 from Scania AB in Sweden. 